\title{
Avaliação de um curso de Odontologia com base nas Diretrizes Curriculares Nacionais: um estudo seccional
}

\author{
Maria Helena Rodrigues Galvão*; Hannah Gil de Farias Morais**; Franklin Delano Soares Forte***; \\ Cláudia Helena Soares de Morais Freitas***; Geraldo Eduardo Guedes de Brito****; Talitha \\ Rodrigues Ribeiro Fernandes Pessoa***
}

\footnotetext{
* Programa de Pós-Graduação em Saúde Coletiva, Universidade Federal do Rio Grande do Norte

** Programa de Pós-Graduação em Ciências Odontológicas, Universidade Federal do Rio Grande do Norte

*** Departamento de Clínica e Odontologia Social, Universidade Federal da Paraíba

**** Departamento de Fisioterapia, Universidade Federal da Paraíba
}

Recebido: 04/10/2021. Aprovado: 03/01/2022.

\begin{abstract}
RESUMO
O estudo teve por objetivo avaliar, a partir da visão de professores e estudantes, um curso de graduação em Odontologia, com base nas Diretrizes Curriculares Nacionais (DCN). Trata-se de um estudo observacional e transversal, com metodologia exploratória, descritiva e de avaliação. A abordagem utilizada foi quantitativa, por meio da aplicação de questionário online, por meio de instrumento com critérios validados. A amostra de professores e estudantes do curso foi censitária. A Integração ensinoserviço obteve o melhor desempenho relativo entre as dimensões avaliadas tanto por estudantes quanto por professores. A maior parte dos estudantes considerou o curso adequado às DCN $(50,2 \%)$, enquanto a maioria dos professores o consideraram parcialmente adequado (56,36\%). Os estudantes mostraram-se mais satisfeitos com a formação ofertada no curso atribuindo maiores valores de média para todas as dimensões e subdimensões. Referente ao perfil do egresso, professores e estudantes atribuíram o pior desempenho à subdimensão "Autônomo", o melhor desempenho foi atribuído às subdimensões "Generalista" e "Capaz de compreender a realidade social". Na dimensão Orientação do cuidado em saúde professores e estudantes atribuíram melhor desempenho à subdimensão "Tratamento odontológico", enquanto o pior desempenho foi atribuído à "Multiprofissionalidade" e "Enfoque epidemiológico". Na dimensão Abordagem pedagógica, o melhor desempenho foi atribuído às subdimensões "Papel do professor" e "Articulação ensino-pesquisa-extensão", o pior desempenho foi atribuído por professores e estudantes à subdimensão "Flexibilização curricular". A dimensão Integração ensino-serviço e subdimensões relacionadas foram bem avaliadas tanto por professores quanto por estudantes. As principais fragilidades encontradas estão relacionadas com dimensões que abordam as relações profissional/paciente e a as relações entre o cirurgião-dentista e a equipe de saúde durante a formação profissional. Faz-se necessária a criação de estratégias de reorientação da formação direcionadas a novos conceitos metodológicos de ensino e aprendizagem para a efetivação das DCN no curso de Odontologia avaliado.
\end{abstract}

Descritores: Educação em Odontologia. Educação Baseada em Competências. Capacitação de Recursos Humanos em Saúde. Currículo. 


\section{INTRODUÇÃO}

$\mathrm{O}$ ensino superior enfrenta grandes mudanças decorrentes de um mundo globalizado. Dentre estas estão as novas formas de relacionamento entre as universidades e a sociedade, o aumento na oferta de vagas e a criação de políticas de inclusão e de acesso ao ensino superior. No caso dos cursos da área da saúde, emerge a necessidade de formação de um novo perfil do egresso, o qual deve contemplar a necessidade de alinhar-se à realidade local, conforme o perfil epidemiológico da população, as complexas relações entre os profissionais de saúde e entre estes e a sociedade ${ }^{1}$.

A formação profissional é um tema em discussão na atualidade em função das mudanças ocorridas pela promulgação da Constituição Federal de 1988 e a proposição do Sistema Único de Saúde (SUS), de forma a considerar as necessidades de saúde da população, os determinantes sociais da saúde e a complexidade do trabalho em saúde ${ }^{2}$.

Dentre os desafios enfrentados pelas instituições formadoras e prestadoras de serviços de saúde, está o da inserção do processo de formação profissional no âmbito dos serviços de saúde. Esta inserção deve ocorrer dentro de uma perspectiva crítica, reflexiva, solidária, colaborativa e construtiva, a fim de que se considerem as transformações no processo ensino aprendizagem, na assistência e no cuidado em saúde ${ }^{1}$.

Historicamente, a prática odontológica se desenvolveu em um contexto privado, com fragmentação de conteúdos e ensino centrado no professor especialista. Esforços foram feitos desde a reforma sanitária para implementação do SUS e para o reordenamento do modelo de prestação da atenção à saúde em todos os níveis de atenção. A inclusão do cirurgião-dentista na atenção básica e a Política Nacional de Saúde Bucal (Brasil Sorridente) representam avanços significativos na reorientação da atenção à saúde bucal no país. Em detrimento disto, a formação profissional ainda carece de uma transformação em razão de continuar sendo orientada pelo antigo modelo biomédico ${ }^{3}$.

As Diretrizes Curriculares Nacionais (DCN) para o curso de Odontologia foram estabelecidas pela Câmara de Educação Superior do Conselho Nacional de Educação e representam um esforço na superação de lacunas na formação superior em Odontologia. As DCN foram formuladas e propostas por núcleos profissionais criados nos anos 2000 e substituíram o antigo currículo mínimo, implantado em $1982^{1}$.

O perfil profissional preconizado pelas DCN por si só se constitui um desafio para a sua prática: perfil do formando em Odontologia generalista, humanista, crítico e reflexivo, para atuar em todos os níveis de atenção à saúde, com base no rigor técnico e científico. O que trouxe para a prática odontológica conceitos sobre ciências sociais e humanas em saúde e a necessidade de focar o exercício profissional de acordo com as necessidades de saúde da população, tanto em nível individual quanto coletivo ${ }^{4}$.

No entanto, após 15 anos de sua implantação, ainda se observam dificuldades na implementação das DCN. Dentre as quais destaca-se a não compreensão adequada destas por grande número de dirigentes, coordenadores, professores e alunos dos cursos de Odontologia do Brasil, o que retarda a sua efetivação direta nos cursos e prejudica as tentativas de revisão das estruturas curriculares das instituições de ensino e o próprio desenvolvimento dos cursos para a formação de um profissional compatível com a realidade das demandas sociais do país ${ }^{5}$. A percepção de tais aspectos e da possibilidade de solucioná-los só é possível mediante processos avaliativos.

Os processos de avaliação foram repensados a partir da Lei de Diretrizes e Bases de 1996. No ano de 2003, o Ministério da Educação propôs o 
Sistema Nacional de Avaliação da Educação Superior (SINAES). E desde então, os cursos de Odontologia e instituições de ensino superior (IES) no Brasil são periodicamente avaliados. Assim, a cultura da avaliação vem sendo progressivamente sendo incorporada e amadurecida na gestão das IES, embora se perceba a necessidade de maior compreensão, elaboração e validação de instrumentos de avaliação que considerem os diversos atores e cenários envolvidos na formação profissional.

A avaliação institucional dos cursos de graduação da área da saúde tem por objetivo conhecer como estes justificam sua existência, a que se propõem, como se estruturam, que processos desenvolvem, que resultados buscam e como efetivamente os alcançam. Nesta direção, a avaliação sistemática, íntegra e eticamente conduzida pode oferecer subsídios que geram discussões, análises e evidências de fragilidades e fortalezas na execução das ações. E que, por sua vez, exigem revisitar o planejamento e as metas institucionais, no realinhamento de estratégias, e no fortalecimento da cultura institucional de avaliação, com a finalidade de alcançar os objetivos propostos na estruturação de tais cursos ${ }^{6}$.

A partir de uma abordagem de pesquisa qualitativa, avaliou-se o currículo de Odontologia de uma IES no sul do Brasil. Foi observado que as mudanças do currículo acontecem em processo, sendo necessário tempo para que tais mudanças sejam implementadas e para que haja uma organização do processo de trabalho. Este processo deve ser firmado em um apoio institucional e direcionado por um grupo efetivo de trabalho. Verificou-se também tensões entre a teoria pensada e a realidade percebida, que podem ser utilizadas em benefício da identificação de lacunas, bem como direcionar os esforços a serem feitos no sentido de alcançar os objetivos inicialmente propostos $^{7}$.

Outra pesquisa, também realizada no Sul do
Brasil, avaliou a mudança do currículo de Odontologia a partir de uma abordagem qualitativa com estudantes. Observou-se a valorização de conteúdos e práticas técnicas da atenção odontológica, como também do reconhecimento do SUS como cenário de aprendizagem importante por parte dos estudantes. O estudo aponta a necessidade da instituição de processos avaliativos contínuos e participativos e de apropriação por parte do coletivo de docentes e discentes do novo currículo proposto, a fim de que ocorra a sua implantação efetiva ${ }^{8}$.

O presente estudo teve por objetivo avaliar, a partir da visão de professores e estudantes, um curso de graduação em Odontologia, utilizando o instrumento desenvolvido e validado por Pessoa e Noro $(2015)^{9}$, verificando a avaliação de professores e estudantes sobre os aspectos relacionados às DCN na formação profissional no curso, além de identificar os fatores potencializadores e dificultadores para a implementação e desenvolvimento das DCN no referido curso.

Após mais de uma década de implantação de um Projeto Pedagógico de Curso (PPC) baseado nas recomendações das DCN, o curso de Odontologia cenário dessa pesquisa vivencia atualmente um processo de reestruturação curricular. Verifica-se a necessidade de avaliação, para que se compreenda os avanços alcançados, os desafios que perduram na implementação das DCN no curso e para que sejam reconduzidos os processos formativos em favor do desenvolvimento de um profissional egresso compatível com o perfil necessário às novas demandas do trabalho em saúde.

\section{MÉTODO}

Trata-se de um estudo seccional, observacional e transversal, com metodologia exploratória, descritiva e de avaliação.

O curso de Odontologia, cenário da pesquisa, 
possui 65 anos de fundação, o currículo atual foi proposto em 2002, com 4.500 horas, integralizado em cinco anos. Participam 96 professores de cinco departamentos de 3 centros de ensino da IES, sediada no nordeste do Brasil.

Foi realizado um censo, com aplicação de questionário a professores e estudantes do curso de Odontologia. A coleta de dados foi realizada entre fevereiro e junho de 2016. Com a finalidade de evitar viés de seleção, foram enviados convites formais para a participação no estudo aos 96 professores e aos 307 estudantes, mediante contato presencial e via correio eletrônico, semanalmente, durante o período de coleta de dados. Para o contato com os estudantes, utilizou-se o espaço de disciplinas regulares de componentes obrigatórios em todos os semestres do curso. O contato com os professores foi individual, quando se solicitava a confirmação do recebimento do questionário e do seu preenchimento.

Com a finalidade de evitar viés de informação, os dados obtidos não continham qualquer identificação dos participantes. Não houve perda de dados, pois a configuração do questionário impedia o preenchimento incompleto.

O critério de inclusão para os professores consistiu na atuação no curso e para os estudantes em estar devidamente matriculado. Estudantes com afastamento por qualquer motivo (doença, mobilidade estudantil ou outros), com matrícula trancada ou que haviam desistido do curso foram excluídos. Professores afastados ou em licença por qualquer motivo também foram excluídos.

O questionário foi aplicado virtualmente, com recursos do serviço gratuito de armazenamento e sincronização de arquivos Google Drive, que armazena atualmente o pacote de aplicativos Google Forms. O questionário aplicado foi o instrumento validado por Pessoa e Noro $(2015)^{9}$. Foi realizado o teste piloto e constada a adequação do instrumento para a população do estudo.
$\mathrm{O}$ instrumento apresenta dimensões, subdimensões e critérios definidores para a avaliação de cursos de Odontologia com base nas DCN. Compreende a avaliação de quatro dimensões, a saber: Perfil do egresso, Orientação do cuidado em saúde, Integração ensino-serviço e Abordagem pedagógica.

Cada dimensão avaliada é dividida em subdimensões, sendo solicitadas notas de 1 a 10 de acordo com a percepção dos participantes para cada subdimensão. Adicionalmente foram elaboradas perguntas para a caracterização dos participantes do estudo.

Para a análise, os dados obtidos foram transferidos para um banco de dados utilizando o pacote estatístico livre GNU PSPP Statistical Analysis Software, versão 0.6.2. no qual foi realizado um processo de conferência, limpeza e codificação.

Os dados de caracterização dos sujeitos foram submetidos à análise descritiva. Para a avaliação das dimensões e subdimensões do questionário, foram adotados os pesos $\mathrm{e}$ pontuações preconizados na validação do instrumento ${ }^{9}$. Para as subdimensões, os valores atribuídos para cada critério foram utilizados assim como coletados dos questionários, variando de 1 a 10, atribuindo valores máximo, mínimo, média, mediana e desvio padrão.

Para a avaliação das quatro dimensões do instrumento, os valores atribuídos para cada subdimensão foram calculados em referência às pontuações máximas definidas para cada critério durante a validação ${ }^{7}$ e então somados, definindo a pontuação de cada dimensão.

Para o cálculo da avaliação geral do curso, a pontuação obtida em cada dimensão foi dividida pelo número respectivo de subdimensões e os valores foram então somados, definindo a pontuação final do curso. Para a avaliação das quatro dimensões e avaliação geral do curso, foram calculadas a média e o desvio padrão e o 
desempenho relativo foi calculado com base na pontuação máxima esperada definida na validação do instrumento. Para cada dimensão isoladamente, assim como para a avaliação geral do curso composta por todas as dimensões, a pontuação final obtida foi referida ao valor máximo possível, o qual corresponde, ao máximo grau de aproximação da imagem-objetivo $\operatorname{adotada}^{10}$ para a formação em
Odontologia preconizada pelas DCN. O desempenho relativo foi calculado por meio da pontuação máxima esperada para cada dimensão e para a avaliação geral do curso. As dimensões, subdimensões e respectivas pontuações máximas para o cálculo do respectivo desempenho e avaliação geral do curso estão apresentadas no quadro I.

Quadro 1. Pontuações máximas para cada critério e dimensão e peso de cada dimensão na avaliação dos cursos de Odontologia

\begin{tabular}{|c|c|c|c|c|}
\hline Dimensão & Subdimensão & $\begin{array}{c}\text { Peso da } \\
\text { subdimensão }\end{array}$ & $\begin{array}{c}\text { Pontuação } \\
\text { máxima da } \\
\text { dimensão }\end{array}$ & $\begin{array}{c}\text { Pontuação } \\
\text { máxima da } \\
\text { dimensão para } \\
\text { avaliação geral }\end{array}$ \\
\hline Perfil do egresso & $\begin{array}{c}\text { Generalista } \\
\text { Humanista } \\
\text { Autônomo } \\
\text { Crítico/reflexivo } \\
\text { Capaz de compreender a } \\
\text { realidade social }\end{array}$ & $\begin{array}{c}10 \\
10 \\
9 \\
8 \\
9\end{array}$ & 46 & 9,2 \\
\hline $\begin{array}{c}\text { Orientação do cuidado } \\
\text { à saúde }\end{array}$ & $\begin{array}{l}\text { Enfoque epidemiológico } \\
\text { Promoção de saúde } \\
\text { Diagnóstico } \\
\text { Tratamento odontológico } \\
\text { Equipe de saúde bucal } \\
\text { Multiprofissionalidade }\end{array}$ & $\begin{array}{c}9 \\
10 \\
8 \\
8 \\
8 \\
8\end{array}$ & 51 & 8,5 \\
\hline $\begin{array}{l}\text { Integração ensino- } \\
\text { serviço }\end{array}$ & $\begin{array}{c}\text { Atividades de estágio } \\
\text { Vivências no SUS }\end{array}$ & $\begin{array}{c}10 \\
9\end{array}$ & 19 & 9,5 \\
\hline $\begin{array}{l}\text { Abordagem } \\
\text { pedagógica }\end{array}$ & $\begin{array}{c}\text { Metodologias ativas } \\
\text { Papel do professor } \\
\text { Desenvolvimento docente } \\
\text { Cenários de aprendizagem } \\
\text { Currículo integrado } \\
\text { Avaliação processual do } \\
\text { aprendizado do aluno } \\
\text { Flexibilização curricular } \\
\text { Articulação ensino- pesquisa- } \\
\text { extensão }\end{array}$ & $\begin{array}{l}10 \\
10 \\
10 \\
10 \\
10 \\
10 \\
\\
10 \\
10\end{array}$ & 80 & 10 \\
\hline \multicolumn{2}{|l|}{ Avaliação geral do curso } & - & - & 37,02 \\
\hline
\end{tabular}

Fonte: Pessoa e Noro, $2015^{9}$ 
O projeto de pesquisa foi aprovado pelo Comitê de Ética institucional, seguindo todas as orientações da Resolução 466/2012 do Conselho

\section{RESULTADOS}

Responderam à solicitação 58 professores em atuação no curso de Odontologia (taxa de resposta de 60,4\%). Dentre estes, 3 professores se recusaram a participar. Considerando os professores que responderam ao questionário online $(n=55)$, observou-se que a maioria $(56,4 \%)$ pertencia ao sexo feminino, com idade acima de 50 anos (40\%), 11 a 20 anos de formação acadêmica, 30,9\% com 11 a 20 anos de exercício da docência, sendo que $90,9 \%$ possuíam o título de doutorado (tabela 1).

No tocante aos estudantes, 201 dos 307 devidamente matriculados no período da coleta de dados responderam à solicitação (taxa de resposta de 65,5\%). Salienta-se que uma parte dos estudantes $(\mathrm{n}=21,6,84 \%)$ se encontrava nos critérios de exclusão do estudo.

A maior parte dos estudantes pertencia ao sexo feminino $(61,7 \%)$, havendo participação de estudantes de todos os períodos. A maior parte dos estudantes referiu que o curso de Odontologia consiste em sua primeira experiência em um curso de graduação $(83,6 \%)$, conforme tabela 2. Quanto à pretensão de inserção no mercado de trabalho, a maior frequência foi atribuída à atuação junto à estratégia de Saúde da Família (31,3\%), como segunda opção, consultório próprio $(26,9 \%)$, a terceira opção foi referente ao ensino e docência (15,9\%).

A tabela 3 apresenta as médias, desvio padrão e o desempenho relativo para as dimensões e para a avaliação geral do curso, considerando as pontuações máximas que poderiam ser alcanças por cada dimensão e pela avaliação geral do curso.
Nacional de Saúde/MS, com Certificado de Apresentação para Apreciação Ética (CAAE) de número 45883815.3.0000.5188.

De modo geral os estudantes avaliaram com médias superiores a maioria das subdimensões, com exceção de "Metodologias ativas" e "Avaliação processual do aprendizado do aluno", embora sem significância estatística. A única exceção foi a subdimensão "Papel do professor", melhor avaliada por professores, com diferença significativa de médias em relação à avaliação dos estudantes nesta subdimensão.

Considerando o desempenho da dimensão Perfil do Egresso, houve diferença estatisticamente significante entre as médias atribuídas por professores e estudantes para a subdimensão "Capaz de compreender a realidade social".

Para a dimensão Orientação do cuidado em saúde houve diferenças estatisticamente significantes entre as médias atribuídas por professores e estudantes para as seguintes subdimensões "Promoção da saúde", "Diagnóstico", "Tratamento Odontológico", "Equipe de saúde bucal" e "Multiprofissionalidade".

$\mathrm{Na}$ dimensão Integração ensino-serviço, os professores atribuíram às subdimensões “Atividades de estágio" e "Vivências no SUS" o mesmo desempenho (média=7,9). Para os estudantes o desempenho das subdimensões foi semelhante, onde a subdimensão "Atividades de Estágio" obteve média 8,1 e "Vivências no SUS" obteve média 8,0.

$\mathrm{Na}$ dimensão Abordagem pedagógica, houve diferenças estatisticamente significantes entre as médias atribuídas por professores e estudantes para a subdimensão "Desenvolvimento docente" $(p=0,000)$. 
Tabela 1. Distribuição das caraterísticas gerais, funcionais e dos componentes curriculares ministrados pelos docentes

\begin{tabular}{|c|c|c|}
\hline Características dos professores & $\mathbf{n}$ & $\%$ \\
\hline \multicolumn{3}{|l|}{ Sexo } \\
\hline Feminino & 31 & 56,4 \\
\hline Masculino & 24 & 43,9 \\
\hline \multicolumn{3}{|l|}{ Faixa etária } \\
\hline 30 a 39 anos & 20 & 36,4 \\
\hline 40 a 49 anos & 13 & 23,6 \\
\hline 50 ou mais anos & 22 & 40 \\
\hline \multicolumn{3}{|l|}{ Tempo de formação } \\
\hline Até 10 anos & 4 & 7,3 \\
\hline 11 a 20 anos & 23 & 48,1 \\
\hline 21 a 30 anos & 13 & 23,6 \\
\hline 31 ou mais anos & 13 & 23,6 \\
\hline Não respondeu & 2 & 3,6 \\
\hline \multicolumn{3}{|l|}{ Titulação } \\
\hline Especialização & 1 & 1,8 \\
\hline Mestrado & 4 & 7,3 \\
\hline Doutorado & 50 & 90,9 \\
\hline \multicolumn{3}{|c|}{ Tempo de exercício da função docente (anos) } \\
\hline 1 a 5 & 4 & 7,3 \\
\hline 6 a 10 & 13 & 23,6 \\
\hline 11 a 20 & 17 & 30,9 \\
\hline 21 a 30 & 14 & 25,5 \\
\hline 31 ou mais & 7 & 12,7 \\
\hline \multicolumn{3}{|c|}{ Desenvolvimento de outras atividades além do ensino } \\
\hline Pesquisa & 46 & 83,6 \\
\hline Extensão & 39 & 70,9 \\
\hline Pós-graduação & 13 & 23,6 \\
\hline Administrativa & 6 & 10,9 \\
\hline \multicolumn{3}{|l|}{ Ano da graduação que leciona } \\
\hline $1^{\mathrm{o}}$ & 9 & 16,4 \\
\hline $2^{\circ}$ & 23 & 41,8 \\
\hline $3^{\circ}$ & 29 & 52,7 \\
\hline $4^{\circ}$ & 20 & 36,4 \\
\hline $5^{\circ}$ & 18 & 32,7 \\
\hline
\end{tabular}


Tabela 2. Distribuição das características dos estudantes de Odontologia participantes

\begin{tabular}{lcc}
\hline Características dos estudantes & $\mathbf{n}$ & $\mathbf{\%}$ \\
\hline Sexo & 124 & 61,7 \\
$\quad$ Feminino & 77 & 38,3 \\
Masculino & & \\
Ano da graduação em curso & 37 & 18,4 \\
$1^{\circ}$ ano & 43 & 21,4 \\
$2^{\circ}$ ano & 47 & 23,4 \\
$3^{\circ}$ ano & 43 & 21,4 \\
$4^{\circ}$ ano & 31 & 15,4 \\
$5^{\circ}$ ano & & \\
Primeira graduação no ensino superior & 168 & 83,6 \\
Odontologia (em curso) & 33 & 16,4 \\
Outros & & \\
Como pretende se inserir no mercado de trabalho & 31,3 & 63 \\
Programa de Saúde da Família & 26,9 & 54 \\
Consultório próprio & 15,9 & 32 \\
Ensino e Docência & 15,4 & 31 \\
Hospitais & 10,4 & 21 \\
Outros *
\end{tabular}

*Pesquisador, Centro de Especialidades Odontológicas, serviço militar, perito odontolegista

A dimensão com melhor avaliação por professores e estudantes foi Integração ensinoserviço com $79,5 \%$ e $80 \%$ do valor máximo esperado para esta dimensão, respectivamente. A dimensão com o menor desempenho segundo a percepção dos professores e estudantes foi Abordagem Pedagógica com desempenho relativo de 70,9\% e 74,9\% respectivamente. Já a Avaliação geral do curso obteve desempenho relativo de $74,4 \%$ para os professores e $78,0 \%$ para os estudantes, considerando a pontuação máxima esperada para o curso graduação nas dimensões avaliadas. Em valores absolutos e variadas proporções, todas as dimensões foram melhor avaliadas por estudantes do que por professores, semelhantemente ao resultado encontrado nas subdimensões, sendo esta diferença significativa para a dimensão Orientação do cuidado em saúde (tabela 4).

\section{DISCUSSÃO}

A avaliação dos cursos de graduação no ensino superior faz-se necessária para o conhecimento de suas estruturas, fundamentos e objetivos. Não deve limitar-se ao ato avaliativo, devendo integrar um processo de avaliação. Este processo revela fragilidades e potencialidades das ações empregadas, levando à reflexão e discussão que norteia a adoção de ações construtivas ${ }^{11}$.

Em um universo de escassez de estudos que propõem uma avaliação dos cursos de saúde com critérios validados, a matriz de critérios desenvolvida por Pessoa e Noro $(2015)^{9}$ traz o diferencial de contar com questões relativas ao perfil do egresso idealizado pelas DCN, bem como questões relativas a demandas atuais de atenção à saúde. Entretanto, percebeu-se como limitação do instrumento a ausência de critérios avaliativos referentes aos aspectos de liderança e gestão. 
A matriz de critérios proposta apresentou confiabilidade de utilização neste estudo (índice de alfa de Cronbach $=0,950$ ). Este índice, que tem variação de 0 a 1 , estima o quão uniformemente os itens contribuem para a soma não ponderada do instrumento. Quanto maior a aproximação do valor do coeficiente de 1 , mais consistente e mais confiável é o instrumento ${ }^{12}$.

De forma geral a opinião dos professores resultou em menores médias, o que pode ser justificado pelo reconhecimento das dificuldades e limitações inerentes à estrutura, organização, processos e perspectivas de mudança da instituição, mesmo que limitadas em suas áreas de atuação. Entretanto, embora os estudantes possuam uma visão menos ampla dos aspectos institucionais estruturantes, estes possuem uma vivência mais abrangente de todos os momentos do curso.

Tabela 3. Avaliação das subdimensões pelos professores e estudantes do curso de Odontologia (Teste de Mann-Whitney)

\begin{tabular}{|c|c|c|c|c|c|c|c|c|}
\hline \multirow{2}{*}{ Dimensões } & \multirow[b]{2}{*}{ Subdimensões } & \multicolumn{3}{|c|}{ Professores } & \multicolumn{3}{|c|}{ Estudantes } & \multirow{2}{*}{$\begin{array}{c}\text { Valor } \\
\text { de } p\end{array}$} \\
\hline & & Média & DP & Mediana & Média & DP & Mediana & \\
\hline \multirow{5}{*}{$\begin{array}{l}\text { Perfil do } \\
\text { egresso }\end{array}$} & Generalista & 7,8 & 1,5 & 8,0 & 8,1 & 1,6 & 8,0 & 0,143 \\
\hline & Humanista & 7,6 & 1,4 & 8,0 & 7,8 & 1,8 & 8,0 & 0,129 \\
\hline & Autônomo & 7,2 & 1,5 & 7,0 & 7,4 & 1,7 & 8,0 & 0,175 \\
\hline & Crítico/reflexivo & 7,4 & 1,6 & 8,0 & 7,8 & 1,5 & 8,0 & 0,056 \\
\hline & $\begin{array}{l}\text { Capaz de compreender a } \\
\text { realidade social }\end{array}$ & 7,4 & 1,6 & 8,0 & 8,2 & 1,6 & 8,0 & $0,001 *$ \\
\hline \multirow{7}{*}{$\begin{array}{l}\text { Orientação } \\
\text { do cuidado } \\
\text { em saúde }\end{array}$} & Enfoque epidemiológico & 7,3 & 1,7 & 8,0 & 7,4 & 1,6 & 8,0 & 0,699 \\
\hline & Promoção da saúde & 7,5 & 1,6 & 8,0 & 8,2 & 1,4 & 8,0 & $0,012 *$ \\
\hline & Diagnóstico & 7,2 & 1,9 & 8,0 & 7,8 & 1,6 & 8,0 & $0,028 *$ \\
\hline & Tratamento & 7,7 & 1,7 & 8,0 & 8,4 & 1,3 & 8,0 & $0,007 *$ \\
\hline & Odontológico & & & & & & & \\
\hline & Equipe de saúde bucal & 6,7 & 2,1 & 7,0 & 7,5 & 1,8 & 8,0 & $0,003 *$ \\
\hline & Multiprofissionalidade & 6,6 & 2,1 & 7,0 & 7,5 & 1,9 & 8,0 & $0,008^{*}$ \\
\hline \multirow{2}{*}{$\begin{array}{l}\text { Integração } \\
\text { ensino- } \\
\text { serviço }\end{array}$} & Atividades de estágio & 7,9 & 1,6 & 8,0 & 8,1 & 1,8 & 9,0 & 0,292 \\
\hline & Vivências no SUS & 7,9 & 1,6 & 8,0 & 8,0 & 1,7 & 8,0 & 0,704 \\
\hline \multirow{8}{*}{$\begin{array}{l}\text { Abordagem } \\
\text { pedagógica }\end{array}$} & Metodologias ativas & 7,2 & 1,6 & 7,0 & 7,4 & 1,7 & 8,0 & 0,278 \\
\hline & Papel do professor & 7,9 & 1,4 & 8,0 & 7,8 & 1,6 & 8,0 & 0,275 \\
\hline & $\begin{array}{l}\text { Desenvolvimento } \\
\text { docente }\end{array}$ & 6,4 & 2,3 & 7,0 & 7,7 & 1,6 & 8,0 & $0,000 *$ \\
\hline & $\begin{array}{l}\text { Cenários de } \\
\text { aprendizagem }\end{array}$ & 7,0 & 2,3 & 8,0 & 7,7 & 1,7 & 8,0 & 0,060 \\
\hline & Currículo integrado & 6,9 & 2,0 & 7,0 & 7,3 & 1,8 & 8,0 & 0,183 \\
\hline & $\begin{array}{l}\text { Avaliação processual do } \\
\text { aprendizado do aluno }\end{array}$ & 7,2 & 1,5 & 7,0 & 7,3 & 1,5 & 7,0 & 0,628 \\
\hline & Flexibilização curricular & 6,3 & 2,1 & 7,0 & 6,7 & 2,0 & 7,0 & 0,304 \\
\hline & $\begin{array}{l}\text { Articulação } \\
\text { ensino/pesquisa/extensão }\end{array}$ & 7,8 & 1,9 & 8,0 & 8,0 & 1,5 & 8,0 & 0,794 \\
\hline
\end{tabular}


Tabela 4. Desempenho relativo, média, desvio padrão e nível de significância (p) da comparação entre médias (Teste de Mann-Whitney) obtido nas dimensões e na avaliação geral do curso pelos professores e estudantes

\begin{tabular}{|c|c|c|c|c|c|c|c|c|}
\hline \multirow[b]{2}{*}{ Dimensões } & \multirow{2}{*}{$\begin{array}{l}\text { Máximo } \\
\text { esperado }\end{array}$} & \multicolumn{3}{|c|}{ Professores } & \multicolumn{3}{|c|}{ Estudantes } & \multirow{2}{*}{$\begin{array}{l}\text { Valor } \\
\text { de } P\end{array}$} \\
\hline & & Média & DP & $\begin{array}{c}\text { Desempenho } \\
\text { relativo }(\%)\end{array}$ & Média & DP & $\begin{array}{l}\text { Desempenho } \\
\text { relativo }(\%)\end{array}$ & \\
\hline $\begin{array}{l}\text { Perfil do } \\
\text { egresso }\end{array}$ & 46 & 34,7 & 6,1 & 75,4 & 36,2 & 5,8 & 78,7 & 0,109 \\
\hline $\begin{array}{l}\text { Orientação } \\
\text { do cuidado } \\
\text { em saúde }\end{array}$ & 51 & 36,5 & 7,6 & 71,6 & 39,7 & 5,8 & 77,8 & $0,012 *$ \\
\hline $\begin{array}{l}\text { Integração } \\
\text { ensino- } \\
\text { serviço }\end{array}$ & 19 & 15,1 & 2,9 & 79,5 & 15,3 & 3,1 & 80,5 & 0,446 \\
\hline $\begin{array}{l}\text { Abordagem } \\
\text { pedagógica }\end{array}$ & 80 & 56,7 & 12,6 & 70,9 & 59,9 & 9,6 & 74,9 & 0,243 \\
\hline $\begin{array}{l}\text { Avaliação } \\
\text { geral do } \\
\text { curso }\end{array}$ & 37,2 & 27,7 & 4,8 & 74,4 & 29,0 & 3,8 & 78,0 & 0,080 \\
\hline
\end{tabular}

Foi observado que na dimensão Perfil do egresso, de uma forma geral, professores avaliaram de forma positiva a subdimensão "Generalista" que define o profissional que no campo coletivo desenvolve ações de promoção de saúde e de gestão de serviços, ao mesmo tempo que no individual, realiza a prevenção, diagnóstico, planejamento e tratamento odontológico dos principais problemas bucais ${ }^{10}$. Já os estudantes avaliaram positivamente a subdimensão "Capaz de compreender a realidade social", ressaltando que estes se enxergam como sujeito que "identifica o contexto social no qual desenvolve sua prática profissional, respeitando as caraterísticas da população e procurando soluções adequadas a esta realidade" .

Os resultados positivos encontrados nessa dimensão remontam a um avanço em direção a uma formação consonante com uma demanda atual, onde os profissionais de saúde devem ser educados para que sejam competentes para atuar de maneira centrada nos pacientes e nas reais necessidades da população. Os profissionais formados nessa lógica devem mobilizar os conhecimentos e habilidades para compor equipes localmente responsivas, cujo objetivo final é garantir a cobertura universal dos serviços abrangentes de alta qualidade que são essenciais para promover oportunidades de equidade em saúde $^{13}$.

$\mathrm{Na}$ dimensão Perfil do egresso, a subdimensão "Autônomo" obteve o menor desempenho tanto para professores como para estudantes. A subdimensão "Autônomo" define o egresso como um profissional que toma decisões tanto em procedimentos clínicos como em situações de gestão e trabalho coletivo com segurança e habilidade ${ }^{9}$. Esse resultado demonstra uma fragilidade do curso no que tange o cumprimento do objetivo das DCN, que é garantir uma capacitação profissional norteada pela autonomia, onde os alunos devem "aprender a aprender", "aprender a ser", "aprender a fazer", "aprender a viver juntos" e "aprender a conhecer". 
O desenvolvimento da autonomia profissional durante os cursos de graduação dialoga com a adoção de metodologias ativas no processo de ensino-aprendizagem. Para que o estudante desenvolva esta habilidade faz-se necessária a inovação, dinamização e problematização de situações, transformando-as em momentos de rico aprendizado que extrapolam os limites de um currículo disciplinar pautado nos valores tradicionais $^{14}$.

Observou-se no curso a preservação da tendência de orientação à saúde conservadora e focada na valorização dos procedimentos odontológicos. Observando a dimensão Orientação do cuidado em saúde, encontramos alguns desafios a serem superados na formação ofertada atualmente no curso de Odontologia. Na percepção dos professores, a maior fragilidade está relacionada a subdimensão "Multiprofissionalidade" que se refere ao desenvolvimento de atividades de orientação ao cuidado multiprofissional e interdisciplinar com alunos, técnicos ou profissionais da mesma área e/ou de outras áreas diferentes, prevendo a integralidade das ações de saúde ${ }^{9}$. Esta dimensão obteve menores médias na avaliação por professores, que se justifica pelo ambiente de aprendizado em Odontologia ser muito restrito às clínicas escolas durante o ciclo profissionalizante, impedindo uma maior interação com outros estudantes e profissionais de áreas afins.

Nestes resultados atribuídos por professores, percebe-se uma forte tendência do modelo de formação tradicional da Odontologia, focado no tratamento odontológico, uma vez que as subdimensões melhores avaliadas tinham relação direta com a resolução dos problemas bucais, enquanto as com menor desempenho se relacionavam com as habilidades de trabalho em equipe.

Os estudantes, por outro lado, percebem como fragilidade na orientação do cuidado em saúde abordada no curso, a subdimensão "Enfoque epidemiológico", não identificando no curso uma "estrutura curricular organizada a partir da realidade epidemiológica da região e do país, com base nas necessidades de saúde da população". Esta subdimensão obteve menor média atribuída por estudantes.

Diante desta conjuntura, fica evidente o esgotamento do processo de formação focado no conceito privatista em Odontologia. As instituições de ensino devem considerar a importância dos novos conceitos em formação, com base nas DCN como um fator diferencial de seus egressos. A humanização, a capacidade de reflexão, a atenção generalista e a compreensão da realidade social devem trazer um novo vigor à classe odontológica, resultando numa maior valorização dos profissionais diante da população.

Um importante achado, relacionado à dimensão Integração ensino-serviço foi a melhor avaliação atribuída por professores e estudantes a esta dimensão. Tal resultado reflete um contraponto à formação tradicional em Odontologia. A melhor avaliação desta dimensão por estudantes nesse ciclo, remonta ao reconhecimento e valorização de tais atividades como importantes processo de formação.

O fortalecimento da Integração ensinoserviço encontrado no curso de Odontologia da instituição do estudo é uma tendência contrária ao que se observa na formação predominante da área odontológica. Em estudo semelhante, que retratou a avaliação dos cursos de Odontologia da região nordeste sob a percepção dos coordenadores de curso, realizado por Pessoa $(2015)^{15}$, a dimensão Integração ensino-serviço obteve o pior desempenho relativo dentre as dimensões avaliadas.

Nesta perspectiva, a principal estratégia colaboradora do processo de mudança de práticas na formação em saúde do curso de Odontologia da instituição em estudo foi a integração ensino- 
serviço, que consistiu em um dos eixos responsáveis pela solidificação da proposta curricular, por meio da implantação de estágios em todos os períodos do curso que representam ações diversas na interface do ensino com o serviço.

Percebeu-se neste estudo, que a maioria dos docentes participantes tinham entre 11 e 20 anos de exercício da função docente, portanto o início de suas carreiras se deu em um contexto totalmente distinto das atuais demandas de formação. Desta forma, torna-se indispensável o estabelecimento de estratégias que possibilitem o desenvolvimento docente, e que tais estratégias não se restrinjam apenas a área de formação em nível técnico clinico procedimental especializado, mas sejam direcionadas principalmente para novos conceitos metodológicos de ensino e aprendizagem.

Outro aspecto importante para a efetivação das DCN no curso de Odontologia avaliado neste estudo, é a subdimensão "Papel do professor" que avaliou o papel do professor como "facilitador da aprendizagem, gestor do conhecimento e articulador de atividades que promovem o aprendizado do aluno",

Em estudo que objetivou analisar os procedimentos de ensino adotados nos cursos de Odontologia, Sena et al. $(2015)^{16}$ verificaram que em $96,7 \%$ dos componentes curriculares analisados adotava-se aulas expositivas como procedimento de ensino; as aulas demonstrativas onde o professor assume o papel de mostrar a aplicação de conceitos foi o segundo procedimento mais encontrado, estando presente em 73,3\% dos componentes curriculares analisados. Um outro estudo conduzido por Chiarella (2015) ${ }^{17}$ avaliou o processo de ensino-aprendizagem de um curso de Medicina por meio de entrevistas com professores, destacando que 56,7\% dos entrevistados referiramse a uma "metodologia bancária de ensino" atribuindo ao seu papel a função de repassar o conhecimento para os estudantes. Tais estudos demonstram uma tendência inversa ao papel do professor requerido pelas DCN, levando os estudantes à uma atitude passiva, acrítica e que apenas reproduz as orientações transmitidas pelo professor.

Com relação à dimensão Abordagem pedagógica, que obteve o menor desempenho relativo na avaliação do curso, destaca-se pelo menor rendimento a subdimensão "Flexibilização curricular" que é definida pela presença de flexibilidade, inclusive de carga horária, no cumprimento do currículo para que os estudantes possam criar caminhos próprios de desenvolvimento de vocações, interesses e potenciais específicos ${ }^{9}$. Esta subdimensão obteve menor média de avaliação por professores e estudantes na dimensão Abordagem pedagógica.

O baixo desempenho na dimensão Flexibilização curricular justifica-se pela elevada carga horária do curso, que além disto é de período integral. Somado a isto, há uma grande quantidade de componentes curriculares, que por sua vez demandam muitas atividades extraclasse, sobretudo nas disciplinas clínicas em que as atividades de limpeza e organização do instrumental e material de consumo demandam muito tempo de dedicação dos estudantes. Não existem muitos espaços nos componentes curriculares para que os estudantes possam sugerir conteúdos de seu interesse e contribuir na construção dos conteúdos ministrados, em contrapartida pela não interação entre os componentes, muitos conteúdos são repassados exaustivamente com repetições em várias disciplinas durante o curso. Este resultado concorda com o encontrado no estudo realizado por Fadel e Baldani $(2013)^{18}$ onde a necessidade de melhor distribuição de carga horária e de conteúdos foi referida por estudantes como uma fragilidade do curso de Odontologia analisado.

Uma importante característica encontrada foi o desempenho da subdimensão "Articulação ensino-pesquisa-extensão" que pode ser descrita 
como o "envolvimento da pesquisa e da extensão como estratégias curriculares que proporcionam formação integral do estudante". Esta subdimensão obteve ótima avaliação por professores e estudantes não estando condicionada a satisfação com o curso, percepção de adequação às DCN e ao ciclo de atuação.

No panorama tradicional, é de se esperar um contexto de desarticulação entre ensino, pesquisa e extensão, onde estas três vertentes se desenvolvem de forma independente, sem gerar impactos transformadores no processo de formação dos estudantes. Neste cenário, o ensino recebe maior destaque e recursos embora tenha pouco enfoque nas necessidades da população, a pesquisa se desenvolve superficialmente sem participação da população ao qual se destina e a extensão se desenvolve à margem da universidade levando à população fragmentos das preocupações universitárias ${ }^{19}$.

Os resultados encontrados vão de encontro a essa perspectiva, indicando que no curso de Odontologia estudado, a articulação ensinopesquisa-extensão ocorre de forma eficaz e contribui para o desenvolvimento do estudante durante a graduação. Faz-se necessária, portanto, a ampliação no número de vagas e bolsas para projetos institucionais existentes, bem como a criação de novos programas que possibilitem mudanças na formação odontológica. Para que a articulação ensino-pesquisa-extensão continue avançando como agente transformador nas práticas de ensino no curso, é imprescindível o repensar da flexibilidade de carga horária que garanta ao estudante a possibilidade de se engajar em projetos extracurriculares.

Uma boa percepção do SUS como local de trabalho, pode ser verificada no desejo dos estudantes se inserirem no âmbito do Programa de Saúde da Família, uma vez que a maioria destes $(31,1 \%)$ referiu-o como primeira opção para a inserção no mercado de trabalho. Uma percepção positiva de estudantes de Odontologia em relação ao SUS também pôde ser verificada no estudo de Noro e Torquato $(2011)^{20}$, onde $80,9 \%$ dos estudantes referiram uma percepção positiva do SUS e $85,1 \%$ consideraram a inserção profissional no SUS provável ou muito provável.

Depreende-se, portanto, que são necessárias mudanças curriculares que ultrapassem a mera organização dos componentes curriculares, as mudanças necessárias devem perpassar pelo arcabouço teórico e pedagógico do curso e pela organização estrutural da instituição de ensino. Para isso, faz-se necessário a qualificação docente, a mudança nos cenários de prática e a abordagem do processo de ensino-aprendizagem ${ }^{21}$. Tais reflexões devem ultrapassar o âmbito ao qual são tradicionalmente circunscritos, a saúde coletiva, e englobar os demais atores envolvidos no processo de ensino com o objetivo de promover uma visão ampliada da formação orientada para o SUS, enfatizando a integralidade nas práticas de saúde ${ }^{22}$.

Compreende-se a necessidade de aprofundar a reflexão sobre os meios e os modos como a formação profissional em Odontologia vem ocorrendo nos cursos de graduação, com o objetivo de verificar se os conteúdos curriculares ministrados e as metodologias de ensino utilizadas oferecem ao estudante o aprendizado tanto dos procedimentos técnicos indispensáveis ao exercício profissional como, também, desenvolvimento de uma visão crítica em relação ao processo de trabalho e ao mundo que o circunda. Não só na qualidade técnica especializada, mas essencialmente da perspectiva científica social, fazendo com que os conceitos aprendidos durante a graduação sejam aplicados à maioria da população, atribuindo maior impacto social às ações de saúde bucal. Para tanto, é fundamental a instituição de estratégias que vão além dos padrões convencionais de educação, repensando as práticas pedagógicas com finalidade de fortalecer a efetivação de mudanças na formação profissional 
em Odontologia 23 .

Ressalta-se a importância do processo avaliativo como um ato contínuo e constante, onde a prática da autoavaliação deve ser estimulada e valorizada nos cursos de graduação. Para o avanço em direção a transformação da formação profissional em saúde, tais processos devem ser capazes de avaliar percepções de todos os atuantes no processo de ensino-aprendizagem visando a qualificação do ensino em saúde.

\section{CONSIDERAÇÕES FINAIS}

Conhecendo as limitações do curso quanto aos critérios avaliados, grande parte dos professores mostraram-se insatisfeitos com a formação ofertada no curso. Ainda assim, a maior parte dos estudantes apresentou-se satisfeita com a formação ofertada no curso e consideraram o curso adequado às DCN, atribuindo maiores médias para a avaliação das dimensões e subdimensões. Embora tenha apresentado muitos avanços desde a última atualização curricular, especialmente no eixo da integração ensino-serviço, o curso de Odontologia da Universidade Federal da Paraíba precisa avançar na efetivação das mudanças propostas pelas DCN. Percebeu-se a necessidade de uma melhor integração curricular, desafio inerente à estrutura do curso, composto por departamentos que possuem formas de atuação distintas, constituindo uma barreira que resulta em uma formação fragmentada.

Foi observado um melhor desempenho da dimensão Integração ensino-serviço, tendo o melhor desempenho relativo entre as dimensões avaliadas. Nesta perspectiva, propõe-se o estabelecimento de uma parceria conjunta entre os departamentos que compõem o curso de Odontologia da instituição estudada.

Finalmente, evidenciou-se a necessidade de melhorias quanto à dimensão abordagem pedagógica e suas subdimensões, concluindo-se que, para a construção de um perfil de egressos em conformidade com as exigências do mercado e com os pré-requisitos contidos nas DCN, deve-se depreender um processo de avaliação dos estudantes que correspondam as habilidades e competências que se deseja construir, o que para tanto é indispensável o estabelecimento de estratégias que possibilitem o desenvolvimento docente, e que tais estratégias não se restrinjam apenas a área de formação a nível de especialização, mas sejam direcionadas principalmente para novos conceitos metodológicos de ensino e aprendizagem.

\section{ABSTRACT \\ Evaluation of a Dentistry course based on the National Curricular Guidelines: a sectional study}

The study had the purpose of evaluating, from the point of view of teachers and students, an undergraduate course in Dentistry, based on the National Curriculum Guidelines (NCG). This is an observational and cross-sectional study, with exploratory, descriptive and evaluative methodology. The approach used was quantitative, through the application of an online survey, by means of an instrument with validated criteria. The sample of teachers and students was a census.

The "Teaching-service Integration" dimension obtained the best relative performance among those evaluated by both students and professors. Most students considered the course adequate to the NCG $(50.2 \%)$, while most professors considered it partially adequate (56.36\%). Students showed themselves to be more satisfied with the training offered in the course, assigning higher average ratings for all dimensions and subdimensions. Regarding the "Graduate Profile", professors and students gave the worst performance to the subdimension "Autonomous"; the best performance was attributed to the subdimensions "Generalist" and "Able to Understand the Social Reality". In the dimension "Healthcare Orientation", both 
teachers and students evaluated the best results in the subdimension "Dental Treatment", while the worst results were attributed to the subdimensions "Multiprofessionalism" and "Epidemiological Approach". In the "Pedagogical Approach" dimension, the best performance was attributed to the subdimensions "Professor's Role" and "Teaching-researchextension Articulation", and the worst performance was attributed by professors and students to the subdimension "Curricular Flexibility". The "Teaching-service Integration" dimension and related subdimensions were well assessed by both professors and students. The main weaknesses found are related to dimensions that address the professional/patient relationships and the relationships between the dental surgeon and the healthcare team during professional qualification. It is necessary to develop strategies for the reorientation of training focused on new methodological concepts of teaching and learning for the implementation of the NCG in the evaluated Dentistry course.

Descriptors: Education, Dental. CompetencyBased Education. Health Human Resource Training. Curriculum.

\section{REFERÊNCIAS}

1. Brasil. Ministério da Educação. Conselho Nacional de Educação. Câmara da Educação Superior. Parecer $n^{\circ} 1.300$ de 06 de novembro de 2001. Aprova as Diretrizes Curriculares Nacionais para os cursos de Farmácia e Odontologia. Diário Oficial União. [internet] 07 Dez 2001. [Acesso em 6 jul. 2021]. Disponível em: http://portal.mec.gov.br/cne/ arquivos/pdf/CES1300.pdf.

2. Brasil. Constituição (1988). Constituição da República Federativa do Brasil. 18.ed. Brasília, DF: Senado, 1988. Lei 8.080, de 19 de setembro de 1990. Dispõe sobre as condições para a promoção, proteção e recuperação da saúde, a organização e o funcionamento dos serviços correspondentes e dá outras providências. Diário Oficial da
União,Brasília, DF, 19 set. 1990a. Seção 1. [Acesso em 4 out. 2021]. Disponível em: http://portal.saude.gov.br/portal/arquivos/pdf/ Lei8142.pdf.

3. Moreira COF, Dias MSA. Diretrizes curriculares na saúde e as mudanças nos modelos de saúde e de educação. ABCS Health Sci. 2015;40(3):46-239.

4. Freitas SFT, Calvo MCM, Lacerda JT. Saúde Coletiva e Novas Diretrizes Curriculares em Odontologia: uma proposta para graduação. Trab Educ Saúde. 2012;10(2):223-34.

5. Morita MC, Kriger L, Carvalho ACP, Haddad AE. Implantação das Diretrizes Curriculares Nacionais em Odontologia. $1^{\circ}$ ed. Maringá: Editora Artes Médicas, 2007, p.160.

6. Matos MS. Análise do perfil dos alunos e da dimensão ético- humanística na formação de cirurgiões-dentistas em dois cursos de odontologia da Bahia. 2006. 286 f. Tese (Doutorado em Educação) - Universidade Federal Da Bahia Faculdade de Educação, Salvador, 2006. [Acesso em 4 de out. de 2021]. Disponível em: http://www. repositorio.ufba.br/ri/handle/ri/10459.

7. Toassi RFC, Stobaus CD, Mosquera JJM, Moyses SJ. Currículo integrado no ensino de Odontologia: novos sentidos para a formação na área da saúde. Interface Comun Saúde Educ. 2012;16(41):529-44.

8. Silveira JLGC, Garcia VL. Mudança curricular em Odontologia: significados a partir dos sujeitos da aprendizagem. nterface Comun Saúde Educ. 2015;19(52):145-58.

9. Pessoa TRRF, Noro LRA. Caminhos para a avaliação da formação em Odontologia: construção de modelo lógico e validação de critérios. Ciênc Saúde Coletiva. 2015;20(7):2277-90.

10. Campos FE, Ferreira JR, Feuerwerker L, Sena RR, Campos JJB, Cordeiro H, et al. Caminhos para aproximar a formação de profissionais de 
saúde das necessidade da atenção básica. Rev Bras Educ Méd. 2001;25(2):53-9.

11. Lampert JB. Avaliação institucional nos cursos de graduação na área de saúde: avaliar o quê e para quê. Cad ABEM. 2009;5(1):4255.

12. Maroco J, Garcia-Marques T. Qual a fiabilidade do alfa de Cronbach?Questões antigas e soluções modernas? Lab Psicol. 2006;4(1):65-90.

13. Frenk J, Chen L, Bhutta ZA, Cohen J, Crisp $\mathrm{N}$, Evans T, et al. Health professionals for a new century: transforming education to strengthen health systems in an interdependent world. Lancet. 2010;376(9756):1923-58.

14. Neta AA, Alves MSCF. A comunidade como local de protagonismo na integração ensinoserviço e atuação multiprofissional. Trab Educ Saúde. 2016;14(1):221-35.

15. Pessoa TRRF. Caminhos para a avaliação da formação em Odontologia: construção de modelo lógico e validação de critérios. Ciênc Saúde Coletiva. 2015;20(7):2277-90.

16. Senna MIB, Ruckert B, Pinto MJB, Lucas SD. Procedimentos de ensino adotados no curso de graduação em Odontologia da Universidade Federal de Minas Gerais: uma análise documental. Arq Odontol, 2015;51(3):12937.

17. Chiarella T, Lima DB, Moura JC, Marques MCC, Marciglia RMG. A Pedagogia de Paulo Freire e o processo ensino aprendizagem na educação médica. Rev Bras Educ Méd.2015; 39(3):418-25.
18. Fadel CB, Baldani MH. Percepções de formandos do curso de Odontologia sobre as Diretrizes Curriculares Nacionais. Trab Educ Saúde. 2013;11(2):339-54.

19. Araujo ME. Palavras e silêncios na educação superior em odontologia. Ciênc Saúde Coletiva. 2006;11(1):179-82.

20. Noro LRA, Torquato SM. Percepção sobre o aprendizado de saúde coletiva e o SUS entre alunos concludentes de curso de odontologia. Trab Educ Saúde. 2010;8(3):43-447.

21. Forte FDS, Pessoa TRRF, Freitas CHSM, Pereira CAL, Junior PMC. Reorientação na formação de cirurgiões-dentistas: o olhar dos preceptores sobre estágios supervisionados no Sistema Único de Saúde (SUS). Interface. 2015;19(supl. 1):831-43.

22. Cavalheiro MTP, Guimarães AL. Formação para o SUS e os desafios da integração ensino serviço. Cad FNEPAS. 2011;1:19-27.

23. Amâncio Filho A. Dilemas e desafios da formação profissional em saúde. Interface Comunic Saúde Educ. 2004;8(15):375-80.

\section{Correspondência para:}

Talitha Rodrigues Ribeiro Fernandes Pessoa e-mail: talitha.ribeiro@yahoo.com.br Universidade Federal da Paraíba - Campus I Departamento de Clínica e Odontologia Social Cidade Universitária 58059-900 João Pessoa/PB 